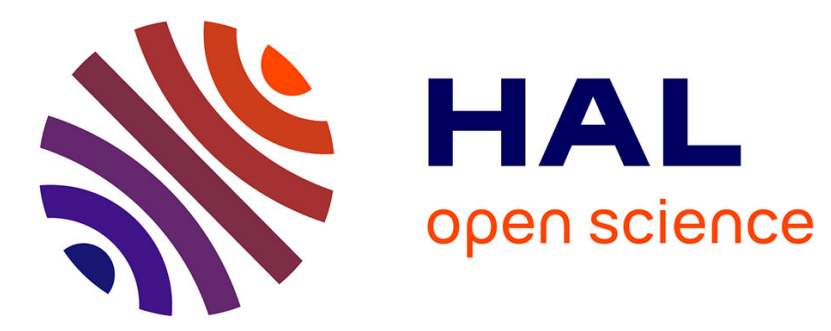

\title{
Semi-algebraic neighborhoods of closed semi-algebraic sets
}

\author{
Nicolas Dutertre
}

\section{To cite this version:}

Nicolas Dutertre. Semi-algebraic neighborhoods of closed semi-algebraic sets. Annales de l'Institut Fourier, 2009, 59 (1), pp.429-458. hal-00018914

\section{HAL Id: hal-00018914 https://hal.science/hal-00018914}

Submitted on 13 Feb 2006

HAL is a multi-disciplinary open access archive for the deposit and dissemination of scientific research documents, whether they are published or not. The documents may come from teaching and research institutions in France or abroad, or from public or private research centers.
L'archive ouverte pluridisciplinaire HAL, est destinée au dépôt et à la diffusion de documents scientifiques de niveau recherche, publiés ou non, émanant des établissements d'enseignement et de recherche français ou étrangers, des laboratoires publics ou privés. 


\title{
SEMI-ALGEBRAIC NEIGHBORHOODS OF CLOSED SEMI-ALGEBRAIC SETS
}

\author{
NICOLAS DUTERTRE
}

\begin{abstract}
Given a closed (not necessarily compact) semi-algebraic set $X$ in $\mathbb{R}^{n}$, we construct a nonnegative semi-algebraic $C^{2}$ function $f$ such that $X=f^{-1}(0)$ and such that for $\delta>0$ sufficiently small, the inclusion $X \subset f^{-1}([0, \delta])$ is a retraction. As a corollary, we obtain several formulas for $\chi(X)$.
\end{abstract}

\section{INTRODUCTION}

Let $X$ be a compact algebraic set in $\mathbb{R}^{n}$. The set $X$ is the set of zeros of a nonnegative polynomial function $f$. This function $f$ may not be proper as it is explained by the following example due to H. King : let $f(x, y)=$ $\left(x^{2}+y^{2}\right)\left(\left(y\left(x^{2}+1\right)-1\right)^{2}+y^{2}\right)$, then $f^{-1}(0)=\{0\}$ but $f\left(x,\left(1+x^{2}\right)^{-1}\right) \rightarrow 0$ as $\|x\| \rightarrow+\infty$.

In [Dur], Durfee proved that any compact algebraic set $X$ can be written as the set of zeros of a proper nonnegative polynomial function $g$. Following's Thom terminology, he called such a function a rug function for $X$. Then he defined the notion of algebraic neighborhoods : a subset $T$ with $X \subset T \subset \mathbb{R}^{n}$ is an algebraic neighborhood of $X$ in $\mathbb{R}^{n}$ if $T=g^{-1}([0, \delta])$, where $g$ is a rug function for $X$ and $\delta$ is a positive real smaller than all nonzero critical values of $g$. Using the gradient vector field of $g$, he showed that the inclusion $X \subset T$ is a homotopy equivalence. Thanks to Lojasiewicz's work [Lo1,Lo2] on the trajectories of a gradient vector field, it is not difficult to see that this homotopy equivalence is actually a retraction. Durfee also proved that two algebraic neighborhoods of a compact algebraic set are isotopic. Here also, this uniqueness result is obtained integrating appropriate gradient vector fields.

If $X$ is a non-compact algebraic set in $\mathbb{R}^{n}$ and $f$ is a nonnegative polynomial such that $X=f^{-1}(0)$, then $X$ is not in general a deformation retract of $f^{-1}([0, \delta])$, where $\delta$ is a small regular value of $f$. Let $f(x, y)=[y(x y-1)]^{2}$ ( $f$ is the square of the Broughton polynomial $[\mathrm{Br}]$ and let $X=f^{-1}(0)$. For $\delta$ a sufficiently small positive regular value of $f, f^{-1}([0, \delta])$ has one connected components whereas $X$ has three.

Our aim is to extend Durfee's results to the case of closed (not necessarily compact) semi-algebraic sets. More precisely, we consider a closed semialgebraic set $X$ in $\mathbb{R}^{n}$ and an open semi-algebraic neighborhood $U$ of $X$

Mathematics Subject Classification (2000) : 14P10, 14P25 . 
in $\mathbb{R}^{n}$. We say that $f: U \rightarrow \mathbb{R}$ is an approaching function for $X$ in $U$ (Definition 2.2) if :

(1) $f$ is semi-algebraic, $C^{2}$, nonnegative,

(2) $X=f^{-1}(0)$,

(3) there exists $\delta>0$ such that $f^{-1}([0, \delta])$ is closed in $\bar{U}$.

However, the notion of approaching function is not enough to get a deformation retract as it is suggested by the Broughton example above. Let $\rho: \mathbb{R}^{n} \rightarrow \mathbb{R}_{\geq 0}$ be a proper $C^{2}$ semi-algebraic function, let $f: U \rightarrow \mathbb{R}$ be a $C^{2}$ nonnegative semi-algebraic function such that $X=f^{-1}(0)$ and let $\Gamma_{f, g}$ be the set of points $x$ in $U \backslash X$ where $\nabla f(x)$ and $\nabla \rho(x)$ are colinear (here $\nabla f$ denotes the gradient vector field of $f$ ). We say that $f$ is $\rho$-quasiregular (Definition 2.5) if there does not exist any sequence $\left(x_{k}\right)_{k \in \mathbb{N}}$ of points in $\Gamma_{f, \rho}$ such that $\left\|x_{k}\right\| \rightarrow+\infty$ and $f\left(x_{k}\right) \rightarrow 0$. A $\rho$-quasiregular approaching semi-algebraic neighborhood of $X$ in $U$ (Definition 3.1) is defined as a set $T=f^{-1}([0, \delta])$ such that :

(1) $f$ is a $\rho$-quasiregular approaching function for $X$ in $U$,

(2) $\delta$ is a positive number smaller than all nonzero critical values of $f$,

(3) $f^{-1}([0, \delta])$ is closed in $\bar{U}$,

(4) $\Gamma_{f, \rho}$ does not intersect $f^{-1}([0, \delta])$ outside a compact subset $K$ of $\mathbb{R}^{n}$.

We prove that $\rho$-quasiregular approaching semi-algebraic neighborhoods always exist (Corollary 2.8) and that if $T=f^{-1}([0, \delta])$ is a $\rho$-quasiregular approaching semi-algebraic neighborhood of $X$ in $U$ then $X$ is a strong deformation retract of $T$ (Theorem 3.2). In order to construct this retraction, we study a vector field $w$ that is equal to the gradient of $f$ inside a compact subset of $\mathbb{R}^{n}$ and to the orthogonal projection of the gradient of $f$ onto the levels of $\rho$ outside a compact set. Using the Lojasiewicz inequality with parameters due to Fekak [Fe] and the usual Lojasiewicz gradient we establish an inequality of "Lojasiewicz's type" for the norm of $w$. The retraction is then achieved "pushing" $T=f^{-1}([0, \delta])$ along the trajectories of $w$.

After we show that two $\rho$-quasiregular approaching semi-algebraic neighborhoods of $X$ are isotopic (Theorem 4.4). As above, the isotopy is obtained integrating a vector field which is equal to a gradient vector field on a compact set of $\mathbb{R}^{n}$ and to the projection of this gradient vector field onto the levels of $\rho$ at infinity.

As a corollary, this enables us to prove that when $X$ is smooth of class $C^{3}$, every approaching semi-algebraic neighborhood of $X$ is isotopic to a tubular neighborhood of $X$ (Theorem 5.7).

We end the paper with degree formulas for the Euler-Poincare characteristic of any closed semi-algebraic set obtained thanks to the machinery developped before (Theorem 6.3, Corollary 6.4 and Corollary 6.5), and with a Petrovskii-Oleinik inequality for the Euler-Poincaré characteristic of any real algebraic set (Proposition 6.8).

The author is very grateful to Zbigniew Szafraniec, Vincent Grandjean, Didier D'Acunto and Andreas Bernig for valuable discussions on this topic. 


\section{2. $\rho$-QUASIREGULAR APPROACHING FUNCTIONS}

In this section, we define the notion of a $\rho$-quasiregular approaching function for a closed semi-algebraic set, which generalizes the notion of a rug function introduced by Durfee [Dur].

Let us consider a closed semi-algebraic set $X$ in $\mathbb{R}^{n}$. Let $U$ be an open semi-algebraic neighborhood of $X$. We know that $X$ is the zero set in $U$ of a continuous nonnegative semi-algebraic function $f: U \rightarrow \mathbb{R}$ (for example one can take for $f$ the restriction to $U$ of the distance function to $X$ ). For any $\delta>0$, the set $f^{-1}([0, \delta])$ is closed in $U$ for the induced topology. However, even if $\delta$ is very small, it is not necessarly closed in $\bar{U}$, as it is shown in the following examples.

Example 1. The set $X=\{0\}$ is a closed semi-algebraic set in $\mathbb{R}$, the set $U=]-1,+\infty$ [ is an open semi-algebraic neighborhood of $X$ in $\mathbb{R}$. Let $f: U \rightarrow \mathbb{R}$ be defined by $f(x)=x^{2}(x+1)$. It is clear that for any $\delta>0$, the set $f^{-1}([0, \delta])$ is not closed in $\bar{U}=[-1,+\infty[$.

Example 2. The set $X=\left\{(x, y) \in \mathbb{R}^{2} \mid y=0\right\}$ is a closed semi-algebraic set in $\mathbb{R}^{2}$, the set $U=\left\{(x, y) \in \mathbb{R}^{2} \mid x^{2} y^{2}<1\right\}$ is an open semi-algebraic neighborhood of $X$ in $\mathbb{R}^{2}$. Let $f: U \rightarrow \mathbb{R}$ be defined by $f(x, y)=y^{2}$. For any $\delta>0$, the set $f^{-1}([0, \delta])$ is not closed in $\bar{U}=\left\{(x, y) \in \mathbb{R}^{2} \mid x^{2} y^{2} \leq 1\right\}$.

We would like to avoid this situation. For this we need to put a condition on the tuple $(X, U, f)$.

Definition 2.1. Let $X$ be a closed semi-algebraic set in $\mathbb{R}^{n}$, let $U$ be an open neighborhood of $X$ and let $f: U \rightarrow \mathbb{R}$ be a nonnegative continuous semialgebraic function such that $X=f^{-1}(0)$. We say that $(X, U, f)$ satisfies the condition $(A)$ if there does not exist any sequence $\left(x_{k}\right)_{k \in \mathbb{N}}$ of points in $U$ such that $\lim _{k \rightarrow+\infty} f\left(x_{k}\right)=0$ and such that $\lim _{k \rightarrow+\infty} x_{k}$ exists and belongs to $\operatorname{Bd}(U)=\bar{U} \backslash U$.

It is clear that this condition is satisfied when $U=\mathbb{R}^{n}$. Let us remark that for any couple $(X, U), X$ being a closed semi-algebraic set in $\mathbb{R}^{n}$ and $U$ an open semi-algebraic neighborhood of $X$, there exists a function $f$ such that $(X, U, f)$ satisfies the condition $(A)$. Let $d_{1}: \mathbb{R}^{n} \rightarrow \mathbb{R}$ be the distance function to $X$ and let $d_{2}: \mathbb{R}^{n} \rightarrow \mathbb{R}$ be the distance function to $\operatorname{Bd}(U)$. Let $d: \mathbb{R}^{n} \rightarrow \mathbb{R}$ be defined by

$$
d(x)=\frac{d_{1}(x)}{d_{1}(x)+d_{2}(x)} .
$$

The tuple $\left(X, U, d_{\mid U}\right)$ satisfies the condition $(A)$.

We will explain how to construct from a function $f$ such that $(X, U, f)$ satisfies the condition $(A)$, a nonnegative continuous semi-algebraic function $g$ such that $X=g^{-1}(0)$ and $g^{-1}([0, \delta])$ is closed in $\bar{U}$ for $\delta$ small enough. Actually we will prove a stronger result.

Let us fix a proper $C^{2}$ semi-algebraic function $\rho: \mathbb{R}^{n} \rightarrow \mathbb{R}_{\geq 0}$. We will denote by $\Sigma_{r}$ the set $\rho^{-1}(r)$, by $D_{r}$ the set $\rho^{-1}([0, r])$ and by $E_{r}$ the set 
$\rho^{-1}\left(\left[r,+\infty[)\right.\right.$. Note that for $r$ sufficiently big, $\Sigma_{r}$ is a non-empty compact $C^{2}$-submanifold of $\mathbb{R}^{n}$. We will call such a $\rho$ a control function.

Lemma 2.2. Let $X$ be a closed semi-algebraic set in $\mathbb{R}^{n}$, let $U$ be an open semi-algebraic neighborhood of $X$ and let $f: U \rightarrow \mathbb{R}$ be continuous nonnegative semi-algebraic function such that $X=f^{-1}(0)$ and $(X, U, f)$ satisfies the condition $(A)$. For every integer $q \geq 0$, let $f_{q}: U \rightarrow \mathbb{R}$ be defined by $f_{q}(x)=(1+\rho(x))^{q} \cdot f(x)$. Let $V \subset U$ be an open semi-algebraic neighborhood of $X$. There exists an integer $q_{0}$ such that for every integer $q \geq q_{0}$, there exists $\delta_{q}>0$ such that $f_{q}^{-1}\left(\left[0, \delta_{q}\right]\right)$ is included in $V$ and closed in $\bar{V}$. Furthermore, if $X$ is compact then one can choose $q_{0}$ such that for every integer $q \geq q_{0}, f_{q}^{-1}\left(\left[0, \delta_{q}\right]\right)$ is compact in $\bar{V}$.

Proof. Let $Z$ be the following closed semi-algebraic set : $Z=\bar{U} \backslash V$. Let $d: \mathbb{R}^{n} \rightarrow \mathbb{R}$ be a continuous nonnegative semi-algebraic function such that $X=d^{-1}(0)$ and $Z=d^{-1}(1)$. Let $U_{1}$ be the open semi-algebraic neighborhood of $X$ in $\mathbb{R}^{n}$ defined by $U_{1}=d^{-1}\left(\left[0, \frac{1}{2}[)\right.\right.$ and let $V_{1}$ be the open semi-algebraic neighborhood of $X$ in $U$ defined by $V_{1}=U_{1} \cap U$. It is straightforward to see that $\overline{V_{1}} \subset V$.

Let us study first the case when $U$ is bounded. There exists $\delta>0$ such that $f^{-1}([0, \delta]) \subset V_{1}$. Otherwise, we would be able to construct a sequence of points $\left(x_{k}\right)_{k \in \mathbb{N}}$ in $\bar{U} \backslash V_{1}$ such that $\lim _{k \rightarrow+\infty} f\left(x_{k}\right)=0$. By compactness of $\bar{U} \backslash V_{1}$, there would exist a subsequence of points $\left(x_{\varphi(k)}\right)_{k \in \mathbb{N}}$ in $\bar{U} \backslash V_{1}$ such that $f\left(x_{\varphi(k)}\right)$ tends to 0 and $x_{\varphi(k)}$ tends to a point $y$ in $\bar{U} \backslash V_{1}$. If $y$ belongs to $U$ then $f(y)=0$, which is impossible. So $y$ belongs to $\bar{U} \backslash U$, which is also impossible by the condition $(A)$. Since $\overline{V_{1}}$ is included in $V$ and $\overline{V_{1}}$ is bounded, the set $f^{-1}([0, \delta])$ is compact in $\bar{V}$.

If $U$ is not bounded and $X$ is not compact, then the following semialgebraic set $F=U \backslash V_{1}$ is unbounded as well. There exists $r_{0}$ such that for every $r \geq r_{0}, \Sigma_{r} \cap F$ is not empty (the set $\left\{r \in \mathbb{R} \mid \Sigma_{r} \cap F \neq \emptyset\right\}$ is an unbounded semi-algebraic set of $\mathbb{R})$. Let $\alpha:\left[r_{0},+\infty[\rightarrow \mathbb{R}\right.$ be defined by

$$
\alpha(r)=\inf \left\{f(x) \mid x \in \Sigma_{r} \cap F\right\} .
$$

The function $\alpha$ is a semi-algebraic function. Let us show that it is positive. If $\alpha(r)=0$ then there exists a sequence of points $\left(x_{k}\right)_{k \in \mathbb{N}}$ in $F \cap \Sigma_{r}$ such that $f\left(x_{k}\right)$ tends to 0 . By compactness of $\Sigma_{r}$, we can extract a subsequence $\left(x_{\varphi(k)}\right)_{k \in \mathbb{N}}$ such that $f\left(x_{\varphi(k)}\right)$ tends to 0 and $x_{\varphi(k)}$ tends to a point $y$ in $\Sigma_{r} \cap \bar{F}$, which is included in $\Sigma_{r} \cap \bar{U}$. If $y$ belongs to $U$ then $f(y)=0$ and so $y$ belongs to $X$, which is impossible for $d(y) \geq \frac{1}{2}$. Hence $y$ is in $\operatorname{Bd}(U)$. This is impossible by the condition $(A)$. The function $\alpha^{-1}$ is semi-algebraic. From Proposition 2.11 in [Co1] (see also Proposition 2.6.1 in [BCR]), there exists $r_{1} \geq r_{0}$ and an integer $q_{0}$ such that $\alpha(r)^{-1}<r^{q}$ for every $r \geq r_{1}$ and every integer $q \geq q_{0}$. This implies that for every $x$ in $F \cap E_{r_{1}}$ and for $q \geq q_{0}, f_{q}(x)=(1+\rho(x))^{q} \cdot f(x)>1$. It is clear that $\left(X, U, f_{q}\right)$ satisfies the condition $(A)$. The same argument as in the case $U$ bounded shows 
that there exists $\epsilon_{q}$ such that $f_{q}^{-1}\left(\left[0, \epsilon_{q}\right]\right) \cap D_{r_{1}}$ is included in $V_{1} \cap D_{r_{1}}$. We take for $\delta_{q}$ the minimum of 1 and $\epsilon_{q}$. Since $\overline{V_{1}} \subset V$, it is easy to see that $f_{q}^{-1}\left(\left[0, \delta_{q}\right]\right)$ is closed in $\bar{V}$.

It remains to study the case $U$ unbounded but $X$ compact. There exists $r_{2}>0$ such that $X \cap E_{r_{2}}$ is empty. Let $\beta:\left[r_{2},+\infty[\rightarrow \mathbb{R}\right.$ be defined by

$$
\beta(r)=\inf \left\{f(x) \mid x \in U \cap \Sigma_{r}\right\} .
$$

Thanks to condition $(A)$, we can prove that it is a positive semi-algebraic function. There exists $r_{3} \geq r_{2}$ and an integer $q_{1}$ such that $\beta(r)^{-1}<r^{q}$ for every $r \geq r_{3}$ and every integer $q \geq q_{1}$. Hence for $x \in U \cap E_{r_{3}}$ and for $q \geq q_{1}$, $f_{q}(x)=(1+\rho(x))^{q} \cdot f(x)>1$. The tuple $\left(X, U, f_{q}\right)$ satisfies the condition $(A)$. As in the previous cases, there exists $\epsilon_{q}>0$ such that $f_{q}^{-1}\left(\left[0, \epsilon_{q}\right]\right) \cap D_{r_{3}}$ is included in $V_{1} \cap D_{r_{3}}$. We take for $\delta_{q}$ the minimum of 1 and $\epsilon_{q}$. The set $f_{q}^{-1}\left(\left[0, \delta_{q}\right]\right)$ is compact in $\overline{V_{1}}$ because it is compact in $\mathbb{R}^{n}$.

Definition 2.3. Let $X$ be a closed semi-algebraic set in $\mathbb{R}^{n}$ and let $U$ be an open semi-algebraic neighborhood of $X$ in $\mathbb{R}^{n}$. A function $f: U \rightarrow \mathbb{R}$ is called an approaching function for $X$ in $U$ if :

(1) $f$ is semi-algebraic, $C^{2}$, nonnegative,

(2) $X=f^{-1}(0)$,

(3) there exists $\delta>0$ such that $f^{-1}([0, \delta])$ is closed in $\bar{U}$. Furthermore if $X$ is compact then $f^{-1}([0, \delta])$ is compact in $\bar{U}$.

Proposition 2.4. Let $X$ be a closed semi-algebraic set in $\mathbb{R}^{n}$ and let $U$ be an open semi-algebraic neighborhood of $X$ in $\mathbb{R}^{n}$. There exist approaching functions for $X$ in $U$.

Proof. From [DM] Corollary C.12, it is possible to find a $C^{2}$ semi-algebraic function $\phi: \mathbb{R}^{n} \rightarrow[0,1]$ such that $X=\phi^{-1}(0)$ and $\operatorname{Bd}(U)=\phi^{-1}(1)$. Let $f$ be the restriction of $\phi$ to $U$. The tuple $(X, U, f)$ satisfies the condition $(A)$. Applying Lemma 2.2 to $f$ and $U$, we can construct approaching functions for $X$ in $U$.

In his study of regularity at infinity of polynomial functions, Tibar introduced the notion of $\rho$-regularity [Ti]. Let us recall this definition in the real setting.

Definition 2.5. Let $f: \mathbb{R}^{n} \rightarrow \mathbb{R}$ be a polynomial function. We say that the fibre $f^{-1}\left(t_{0}\right)$ is $\rho$-regular at infinity if for any sequence $\left(x_{k}\right) \subset \mathbb{R}^{n}$ such that $\left\|x_{k}\right\| \rightarrow+\infty$ and $f\left(x_{k}\right) \rightarrow t_{0}$, there exists $k_{0}$ such that if $k \geq k_{0}$, then $f^{-1}\left(f\left(x_{k}\right)\right)$ is transverse to $\rho^{-1}\left(\rho\left(x_{k}\right)\right)$ at $x_{k}$.

Tibar proved that if the fibre $f^{-1}\left(t_{0}\right)$ is $\rho$-regular at infinity then $f$ is topologically trivial at infinity at $t_{0}$. As a consequence, if an interval $[a, b]$ does not contain any critical value of $f$ and is such that every fibre $f^{-1}(t)$, $t \in[a, b]$, is $\rho$-regular at infinity, then $f$ is topologically trivial over $[a, b]$.

We will need a slight modification of this definition. For every open semialgebraic set $U$ and for every $C^{2}$ semi-algebraic function $g: U \rightarrow \mathbb{R}$, let $\Gamma_{g, \rho}$ 
be the semi-algebraic set defined by :

$$
\Gamma_{g, \rho}=\{x \in U \mid \nabla g(x) \text { and } \nabla \rho(x) \text { are colinear and } g(x) \neq 0\} .
$$

Definition 2.6. Let $g: U \rightarrow \mathbb{R}$ be a $C^{2}$ semi-algebraic function. We say that $g$ is $\rho$-quasiregular if there does not exist any sequence $\left(x_{k}\right)_{k \in \mathbb{N}}$ in $\Gamma_{f, \rho}$ such that $\left\|x_{k}\right\|$ tends to infinity and $\left|g\left(x_{k}\right)\right|$ tends to 0 .

Note that our definition does not imply that $g^{-1}(0)$ has only isolated singularities, unlike Tibar's definition.

Proposition 2.7. Let $X$ be a closed semi-algebraic set in $\mathbb{R}^{n}$ and let $U$ be an open semi-algebraic neighborhood of $X$. Let $f: U \rightarrow \mathbb{R}$ be a $C^{2}$ semialgebraic nonnegative function such that $X=f^{-1}(0)$. For every integer $q$, let $f_{q}: U \rightarrow \mathbb{R}$ be defined by $f_{q}=(1+\rho(x))^{q} . f(x)$. There exists an integer $q_{0}$ such that for every integer $q \geq q_{0}$, the function $f_{q}$ is $\rho$-quasiregular.

Proof. Let $r_{0}$ be the greatest critical value of $\rho$ and let $\left.\beta:\right] r_{0},+\infty[\rightarrow \mathbb{R}$ be defined by

$$
\beta(r)=\inf \left\{f(x) \mid x \in \Sigma_{r} \cap \Gamma_{f, \rho}\right\} .
$$

The function $\beta$ is semi-algebraic. It is positive since for $r>r_{0}$, the function $f_{\mid \Sigma_{r} \cap U}$ admits a finite number of critical values. As in Lemma 2.2, this implies that there exists $r_{1}>r_{0}$ and an integer $q_{0}$ such that for $x \in \Gamma_{f, \rho} \cap E_{r_{1}}$ and for $q \geq q_{0},(1+\rho(x))^{q} . f(x)>1$. Since $\Gamma_{f, \rho}=\Gamma_{f_{q}, \rho}$, every function $f_{q}$ is $\rho$-quasiregular for $q \geq q_{0}$.

Corollary 2.8. Let $X$ be a closed semi-algebraic set in $\mathbb{R}^{n}$ and let $U$ be an open semi-algebraic neighborhood of $X$. Let $f: U \rightarrow \mathbb{R}$ be a $C^{2}$ semialgebraic nonnegative function such that $X=f^{-1}(0)$. Assume that $(X, U, f)$ satisfies the condition $(A)$. For every integer $q \geq 0$, let $f_{q}: \mathbb{R}^{n} \rightarrow \mathbb{R}$ be defined by $f_{q}(x)=(1+\rho(x))^{q} . f(x)$. There exists an integer $q_{0}$ such that for every $q \geq q_{0}$, the function is a $\rho$-quasiregular approaching function for $X$ in $U$.

If $X$ is an algebraic set, it is the zero set of a nonnegative polynomial $f$. Choosing for $\rho$ a proper nonnegative polynomial and applying the above process, we obtain $\rho$-quasiregular approaching functions for $X$ that are nonnegative polynomials.

Let us compare our notion of $\rho$-quasiregular approaching function with the notion of rug function due to Durfee [Dur]. If $X$ is a compact algebraic set of $\mathbb{R}^{n}$, a rug function for $X$ is a proper nonnegative polynomial $f$ such that $X=f^{-1}(0)$. It is clear that such a function is a $\rho$-quasiregualr approaching function for $X$ in $\mathbb{R}^{n}$.

\section{Retraction on a Closed Semi-Algebraic Set}

In this section, we prove that any closed semi-algebraic set is a strong deformation retract of certain closed semi-algebraic neighborhoods of it. First let us specify the closed semi-algebraic neighborhoods that we will consider. 
Definition 3.1. Let $X \subset \mathbb{R}^{n}$ be a closed semi-algebraic set, let $\rho$ be a control function and let $U$ be an open semi-algebraic neighborhood of $X$. A subset $T$ with $X \subset T \subset U$ is a $\rho$-quasiregular approaching semi-algebraic neighborhood of $X$ in $U$ if $T=f^{-1}([0, \delta])$ where :

(1) $f$ is a $\rho$-quasiregular approaching function for $X$ in $U$,

(2) $\delta$ is a positive number smaller than all nonzero critical values of $f$,

(3) $f^{-1}([0, \delta])$ is closed in $\bar{U}$ and compact in $\bar{U}$ if $X$ is compact,

(4) if $\Gamma_{f, \rho}$ is the following polar set:

$\Gamma_{f, \rho}=\{x \in U \backslash X \mid \nabla f(x)$ and $\nabla \rho(x)$ are colinear $\}$, then $\Gamma_{f, \rho}$ does not intersect $f^{-1}([0, \delta])$ outside a compact subset $K$ of $\mathbb{R}^{n}$.

For short, we will say that such a $T$ is an approaching semi-algebraic neighborhood. By the results of the previous section, it is clear that approaching semi-algebraic neighborhoods always exist.

Theorem 3.2. Let $X$ be a closed semi-algebraic set and let $T$ be an approaching semi-algebraic neighborhood of $X$. Then $X$ is a strong deformation retract of $T$.

Proof. If $X$ is compact, this is already proved by Durfee [Du] and Lojaziewicz [Lo1,Lo2]. So let us assume that $X$ is not compact.

Let us fix $f, U, \delta, \rho$ and $K$ which satisfy the conditions of the above definition and such that $T=f^{-1}([0, \delta])$. Furthermore let us assume that $\delta<1$. We will focus first on the behaviour of $f$ at infinity.

Let $r_{0}>0$ be such that $K \cap E_{r_{0}}$ is empty and such that $\Sigma_{r}$ is a $C^{2}$ submanifold for $r \geq r_{0}$. Let $A=T \cap E_{r_{0}}$. The set $A$ is a closed semialgebraic set of $\mathbb{R}^{n}$ and $A \cap \Gamma_{f, \rho}$ is empty. Let us consider the following closed semi-algebraic set $Y$ of $\mathbb{R}^{n+1}$ :

$$
Y=\left\{(x, t) \in \mathbb{R}^{n+1} \mid x \in A \text { and } \rho(x)=t\right\} .
$$

We will denote by $Y_{t}$ the fibre $\{x \in A \mid(x, t) \in Y\}$. Observe that $Y_{t}=A \cap \Sigma_{t}$. Let $F: A \rightarrow \mathbb{R}$ be defined by :

$$
F(x)=\left\|\nabla f(x)-\left\langle\nabla f(x), \frac{\nabla \rho(x)}{\|\nabla \rho(x)\|}\right\rangle \cdot \frac{\nabla \rho(x)}{\|\nabla \rho(x)\|}\right\| .
$$

The function $F$ is just the norm of the orthogonal projection of $\nabla f(x)$ on the manifold $\Sigma_{\rho(x)}$. Moreover it is a continuous semi-algebraic function on $A$. Let $\tilde{f}$ and $\tilde{F}$ be the semi-algebraic functions defined on $Y$ by $\tilde{f}(x, t)=f(x)$ and $\tilde{F}(x, t)=F(x)$. They are continuous in $x$ and verify $\tilde{F}^{-1}(0) \subset \tilde{f}^{-1}(0)$. This inclusion is easy to check since $F(x)=0$ if and only if $\nabla f(x)$ and $\nabla \rho(x)$ are colinear. On $A$, this can occur only if $x$ belongs to $X$.

We can apply Lojasiewicz's inequality with parameters due to Fekak (see [Fe], p128). We need some notations : for every $t, \tilde{f}_{t}$ and $\tilde{F}_{t}$ are the functions on $Y_{t}$ defined by $\tilde{f}_{t}(x)=\tilde{f}(x, t)$ and $\tilde{F}_{t}(x)=\tilde{F}(x, t)$; for every $S \subset \mathbb{R}, Y_{S}$ denotes the set $Y \cap\left(\mathbb{R}^{n} \times S\right)$. Fekak's theorem states that there exists a finite 
partition into semi-algebraic subsets of $\mathbb{R}=\cup S_{i}$, continuous semi-algebraic functions $h_{i}: Y_{\mid S_{i}} \rightarrow \mathbb{R}$ and rationnal numbers $p_{i} / q_{i}$ such that :

i) $|\tilde{f}(x, t)|^{p_{i} / q_{i}} \leq h_{i}(x, t)|\tilde{F}(x, t)|$ on $Y_{\mid S_{i}}$ for $t \in S_{i}$,

ii) $p_{i} / q_{i}$ is the Lojasiewicz exponent of $\tilde{f}_{t}$ with respect to $\tilde{F}_{t}$ for $t \in S_{i}$. Since $\cup S_{i}$ is a finite semi-algebraic partition of $\mathbb{R}$, there exist $t_{0} \in \mathbb{R}$ and $i_{0}$ such that $S_{i_{0}}=\left[t_{0},+\infty\left[\right.\right.$. Then for every $t \geq t_{0}$, we have:

i) $|\tilde{f}(x, t)|^{p_{i} / q_{i}} \leq h_{i}(x, t)|\tilde{F}(x, t)|$ for $x \in Y_{t}$,

ii) $p_{i_{0}} / q_{i_{0}}$ is the Lojasiewicz exponent of $\tilde{f}_{t}$ with respect to $\tilde{F}_{t}$.

We know that $\tilde{f}_{t}=f_{\mid Y_{t}}$ and $\tilde{F}_{t}=\left\|\nabla\left(f_{\mid Y_{t}}\right)\right\|$. By Lojasiewicz's gradient inequality applied to $f_{\mid Y_{t}}$, we get $p_{i_{0}} / q_{i_{0}}<1$. Let $\alpha=p_{i_{0}} / q_{i_{0}}$ and let $B=T \cap E_{t_{0}}$. We have proved that there exist $0 \leq \alpha<1$ and a continuous semi-algebraic function $h: B \times\left[t_{0},+\infty[\rightarrow \mathbb{R}\right.$ such that for every $x \in B$ :

$$
|f(x)|^{\alpha} \leq h(x, \rho(x)) \cdot F(x),
$$

where $F(x)$ is the norm of the vector field

$$
v(x)=\nabla f(x)-\left\langle\nabla f(x), \frac{\nabla \rho(x)}{\|\nabla \rho(x)\|}\right\rangle \cdot \frac{\nabla \rho(x)}{\|\nabla \rho(x)\|} .
$$

Let $C$ be the compact semi-algebraic set defined by $C=T \cap D_{2 t_{0}}$. By the Lojasiewicz gradient inequality, there exits $d>0$ and $0 \leq \beta<1$ such that on $C$ :

$$
|f(x)|^{\beta} \leq d .\|\nabla f(x)\| .
$$

Here we have applied the Kurdyka-Parusinski version of the Lojasiewicz gradient inequality $[\mathrm{KP}]$.

We will glue the two vector fields $v$ and $\nabla f$. Let $\varphi: \mathbb{R}^{n} \rightarrow \mathbb{R}$ be a $C^{\infty}$-function such that :

- $\varphi(x)=1$ if $\rho(x) \leq 1,3 t_{0}$,

- $\varphi(x)=0$ if $\rho(x) \geq 1,7 t_{0}$,

- $0<\varphi(x)<1$ if $1,3 t_{0}<\rho(x)<1,7 t_{0}$.

Let $w$ be the following vector field on $T$ :

$$
w(x)=(1-\varphi(x)) v(x)+\varphi(x) \nabla f(x) .
$$

We want to find an inequality of "Lojasiewicz's type" for $\|w\|$. First observe that $\|w(x)\| \geq\|v(x)\|$, for

$$
w(x)=v(x)+\varphi(x) \cdot\left\langle\nabla f(x), \frac{\nabla \rho(x)}{\|\nabla \rho(x)\|}\right\rangle \cdot \frac{\nabla \rho(x)}{\|\nabla \rho(x)\|} .
$$

Let $M$ be defined by :

$$
M=\max \left\{h(x, \rho(x)) \mid x \in T \text { and } 1,2 t_{0} \leq \rho(x) \leq 1,8 t_{0}\right\} .
$$

We have $|f(x)|^{\alpha} \leq M .\|w(x)\|$ for $x \in T \cap\left\{x \mid 1,2 t_{0} \leq \rho(x) \leq 1,8 t_{0}\right\}$. For $x \in T \cap D_{1,3 t_{0}},|f(x)|^{\beta} \leq d .\|\nabla f(x)\|$ and $\nabla f(x)=w(x)$. Calling $\gamma$ the 
maximum of $\alpha$ and $\beta$ and $N$ the maximum of $M$ and $d$ and since $\delta<1$, we get that for $x \in T \cap D_{1,8 t_{0}}$ :

$$
|f(x)|^{\gamma} \leq N .\|w(x)\| .
$$

Now for $x \in T \cap E_{1,7 t_{0}}, w(x)=v(x)$ and then :

$$
\left.|f(x)|^{\gamma} \leq h(x, \rho(x))\right) \cdot\|w(x)\| .
$$

On one hand, we have :

$$
\langle\nabla f(x), w(x)\rangle=(1-\varphi(x))\langle\nabla f(x), v(x)\rangle+\varphi(x)\langle\nabla f(x), \nabla f(x)\rangle,
$$

hence

$$
\langle\nabla f(x), w(x)\rangle=(1-\varphi(x))\langle v(x), v(x)\rangle+\varphi(x)\langle\nabla f(x), \nabla f(x)\rangle,
$$

since $\langle v(x), \nabla f(x)\rangle=\langle v(x), v(x)\rangle$. On the other hand,

$$
\langle w(x), w(x)\rangle=\left(1-\varphi(x)^{2}\right)\langle v(x), v(x)\rangle+\varphi(x)^{2}\langle\nabla f(x), \nabla f(x)\rangle .
$$

Using the fact that $0 \leq \varphi(x) \leq 1$, it is easy to see that

$$
\langle\nabla f(x), w(x)\rangle \geq\langle w(x), w(x)\rangle \Leftrightarrow\langle\nabla f(x), \nabla f(x)\rangle \geq\langle v(x), v(x)\rangle .
$$

Since the inequality on the right hand side is verified, we have proved :

$$
\langle\nabla f(x), w(x)\rangle \geq\langle w(x), w(x)\rangle .
$$

We are going to integrate the vector field $-\frac{w}{\|w\|}$. Let $\phi_{t}$ be the flow associated with the differential equation :

$$
\dot{x}=-\frac{w}{\|w\|} .
$$

For every $x \in T$, let $b(x)=\sup \left\{t \mid f\left(\phi_{t}(x)\right) \geq 0\right\}$ and $\omega(x)=\lim _{t \rightarrow b(x)} \phi_{t}(x)$. We write $\phi_{x}(t)$ the trajectory that passes through $x$. The following facts are proved using inequalities (1), (2) and (3) and adapting to our situation the techniques of Lojasiewicz (see [Lo1], [Lo2], [Ku], [KMP] or [NS] for details).

Fact 1 For all $x \in T,\left\{\phi_{x}(t) \mid 0 \leq t \leq b(x)\right\} \subset T$.

Fact 2 For all $x \in T \cap E_{1,7 t_{0}}$, for all $t$ such that $0 \leq t \leq b(x),\left\|\phi_{x}(t)\right\|=$ $\|x\|$.

Fact 3 For all $x \in T \cap D_{1,8 t_{0}}$, for all $t$ such that $0 \leq t \leq b(x),\left\|\phi_{x}(t)\right\| \leq$ $1,8 t_{0}$.

Fact 4 For all $x \in T, b(x)<+\infty$.

Fact 5 For all $x \in T, f(\omega(x))=0$.

Fact 6 The mapping $\omega: T \rightarrow X, x \mapsto \omega(x)$ is continuous.

Fact 7 The mapping $b: T \rightarrow X, x \mapsto b(x)$ is continuous. 
Now we can end the proof of Theorem 3.2. The retraction is given by the following mapping :

$$
\begin{aligned}
G:[0,1] \times T & \rightarrow & T \\
(t, x) & \mapsto & G(t, x)=\phi(t b(x), x) .
\end{aligned}
$$

If $\delta \geq 1$, we can push $f^{-1}([0, \delta])$ onto $f^{-1}\left(\left[0, \delta^{\prime}\right]\right), \delta^{\prime}<1$, along the trajectories of $V$.

We end this section with a remark. Using the same method, one can prove the following result. Let $X \subset \mathbb{R}^{n}$ be a closed semi-algebraic set and let $f: \mathbb{R}^{n} \rightarrow \mathbb{R}$ be a nonnegative semi-algebraic function such that $X=f^{-1}(0)$. Let $\Gamma_{f, \rho}$ be the set defined by

$$
\Gamma_{f, \rho}=\left\{x \in \mathbb{R}^{n} \mid \nabla f(x) \text { and } \nabla \rho(x) \text { are not colinear and } f(x) \neq 0\right\} .
$$

Let $r$ be a regular value of $\rho$. Assume that the following assumption is satisfied : there is no sequence of points $\left(x_{k}\right)$ in $\Gamma_{f, \rho} \cap D_{r}$ such that $\rho\left(x_{k}\right) \rightarrow r$ and $f\left(x_{k}\right) \rightarrow 0$. Then for $\delta>0$ sufficiently small, the inclusion $X \cap D_{r} \subset$ $f^{-1}([0, \delta]) \cap D_{r}$ is a deformation retract.

For example, this result can be applied if $f$ has only isolated critical points on its zero level and $X$ intersects $\Sigma_{r}$ transversally.

\section{UNIQUENESS OF $\rho$-QUASIREGULAR APPROACHING NEIGHBORHOOD}

In this section, we prove that two $\rho$-quasiregular approaching semi-algebraic neighborhoods of a closed non-compact semi-algebraic set are isotopic. Let us recall what Durfee proved in the case of a compact semi-algebraic set.

Theorem 4.1. Let $X$ be a compact semi-algebraic set, let $f_{1}$ and $f_{2}$ be two rug functions for $X$. Let $\delta_{1}$ (resp. $\delta_{2}$ ) be a positive regular value of $f_{1}$ (resp. $\left.f_{2}\right)$ smaller than all nonzero critical values of $f_{1}$ (resp. $\left.f_{2}\right)$. Let $T_{1}=f_{1}^{-1}\left(\left[0, \delta_{1}\right]\right)$ and $T_{2}=f_{2}^{-1}\left(\left[0, \delta_{2}\right]\right)$. There is a continuous family of homeomorphisms $h_{t}: \mathbb{R}^{n} \rightarrow \mathbb{R}^{n}, 0 \leq t \leq 1$, such that :

(1) $h_{0}$ is the identity,

(2) for all $t, h_{t \mid X}$ is the identity,

(3) $h_{1}\left(T_{1}\right)=T_{2}$ and $h_{1}$ is a smooth diffeomorphism of $T_{1} \backslash X$ onto $T_{2} \backslash X$.

Proof. See [Dur], Proposition 1.7. Actually Durfee considers the case of a compact algebraic set but his proof works in our case.

We will prove the following theorem.

Theorem 4.2. Let $X$ be a closed non-compact semi-algebraic set and let $\rho$ be a control function. If $T_{1}$ and $T_{2}$ are two $\rho$-quasiregular approaching semi-algebraic neighborhoods of $X$ in $U$ then there is a continuous family of homeomorphisms $h_{t}: \mathbb{R}^{n} \rightarrow \mathbb{R}^{n}, 0 \leq t \leq 1$, such that :

(1) $h_{0}$ is the identity,

(2) for all $t, h_{t \mid X}$ is the identity,

(3) $h_{1}\left(T_{1}\right)=T_{2}$ and $h_{1}$ is a smooth diffeomorphism of $T_{1} \backslash X$ onto $T_{2} \backslash X$. 
Proof. Let us write $T_{i}=f_{i}^{-1}\left(\left[0, \delta_{i}\right]\right)$ where $f_{i}$ is a $\rho$-quasiregular approaching function for $X$ in $U_{i}, i=1,2$. We will prove our result adapting the ideas of Durfee [Dur]. There are three steps.

Let us first consider the case $f_{1}=f_{2}=f$ and $U_{1}=U_{2}=U$. We can assume without loss of generality that $\delta_{1}<\delta_{2}$. Thanks to condition (4) in Definition 3.1, we see that $f^{-1}(\delta)$ is $\rho$-regular at infinity for every $\delta$ in $\left[\delta_{1}, \delta_{2}\right]$. Since $\left[\delta_{1}, \delta_{2}\right]$ does not contain any critical value of $f$, Tibar's work implies that $T_{1}$ and $T_{2}$ are diffeomorphic. Let us be more precise and explain how the family $h_{t}$ is obtained. As we did in the proof of Theorem 3.2, we can construct a vector field $w$ on $f^{-1}\left(\left[\delta_{1}, \delta_{2}\right]\right)$ which is equal to the orthogonal projection of $\nabla f$ on the levels of $\rho$ outside a set $D_{R}$, and equal to $\nabla f$ inside a set $D_{R^{\prime}}, R^{\prime}<R$. Then we extend $w$ to a complete vector field $\tilde{w}$ defined on $\mathbb{R}^{n}$ using a smooth function equal to 1 on the closed set $f^{-1}\left(\left[\delta_{1}, \delta_{2}\right]\right)$ and to 0 on the closed set $X \cup\left(\mathbb{R}^{n} \backslash U\right)$. Integrating this vector field gives the required family $h_{t}$.

The second case is when $f_{2}=(1+\rho)^{q} f_{1}$ and $U_{1}=U_{2}=U$. Let $\delta$ be the minimum of $\delta_{1}$ and $\delta_{2}$. Let $v_{1}$ (resp. $v_{2}$ ) be the orthogonal projection of $\nabla f_{1}$ on the levels of $\rho$. By condition (4) in Definition 3.1, there exists $R>0$ such that $v_{1}$ and $v_{2}$ do not vanish in $\left.\left.f_{1}^{-1}(] 0, \delta\right]\right) \cap E_{R}$. It is clear that on this set, they do not point in opposite direction. There exists a neighborhood $U^{\prime}$ of $X \cap D_{2 R}$ in $D_{2 R}$ such that $\nabla f_{1}$ and $\nabla f_{2}$ are nonzero and do not point in opposite direction on $U^{\prime} \backslash X$. This fact is proved in the same way as Lemma 1.8 in [Dur]. Hence there exists $\delta^{\prime}$ such that $\nabla f_{1}$ and $\nabla f_{2}$ are nonzero and do not point in opposite direction on $\left.\left.f_{1}^{-1}(] 0, \delta^{\prime}\right]\right) \cap D_{2 R}$. Let $\delta$ " be the minimum of $\delta$ and $\delta^{\prime}$. By the first case, it is enough to prove that $f_{2}\left(\left[0, \delta^{\prime \prime}\right]\right)$ and $f_{1}\left(\left[0, \delta^{\prime \prime}\right]\right)$ are isotopic. Let $S$ be the closed set $f_{1}^{-1}\left(\left[0, \delta^{\prime \prime}\right]\right) \backslash f_{2}^{-1}\left(\left[0, \delta^{\prime \prime}[)\right.\right.$ and let $g: S \rightarrow[0,1]$ be defined by :

$$
g(x)=\frac{f_{2}(x)-\delta "}{f_{2}(x)-f_{1}(x)} .
$$

Note that $g^{-1}(0)=f_{2}^{-1}\left(\delta^{\prime \prime}\right)$ and $g^{-1}(1)=f_{1}^{-1}\left(\delta^{\prime \prime}\right)$. The gradient of $g$ is

$$
\nabla g(x)=\frac{\left(f_{2}(x)-\delta "\right) \nabla f_{1}(x)+\left(\delta^{\prime \prime}-f_{1}(x)\right) \nabla f_{2}(x)}{\left(f_{2}(x)-f_{1}(x)\right)^{2}} .
$$

Let $v$ be its orthogonal projection on the levels of $\rho$. It is nonzero in $S \cap E_{R}$. Moreover, $\nabla g$ is nonzero in $S \cap D_{2 R}$. Gluing these two vector fields, we obtain a $C^{1}$ vector field $w$ on $S$ and we proceed as in the first case.

The third case is the general case. Let $U=U_{1} \cap U_{2}$. By Lemma 2.2 and the second case above, we can assume that $T_{1} \subset U, T_{2} \subset U$ and $T_{1}$ and $T_{2}$ are closed in $\bar{U}$. We need some lemmas.

Lemma 4.3. For every integer $q \geq 0$, let $f_{1 q}: \mathbb{R}^{n} \rightarrow \mathbb{R}$ be defined by $f_{1 q}(x)=(1+\rho(x))^{q} \cdot f_{1}(x)$. Let $v_{1 q}$ (resp. $\left.v_{2}\right)$ be the orthogonal projection of $\nabla f_{1 q}\left(\right.$ resp $\left.\nabla f_{2}\right)$ on the levels of $\rho$. There exist $q_{0} \in \mathbb{N}$ and $R>0$ such that for all $q \geq q_{0}$ the vector fields $v_{1 q}$ and $v_{2}$ are nonzero and do not point in 
opposite direction in $\left.\left.f_{1 q}^{-1}(] 0, \delta_{q}\right]\right) \cap E_{R}$, where $\delta_{q}$ is a small regular value of $f_{1 q}$ such that $f_{1 q}^{-1}\left(\left[0, \delta_{q}\right]\right) \subset U$ and $f_{1 q}^{-1}\left(\left[0, \delta_{q}\right]\right)$ is closed in $\bar{U}$.

Proof. We known that there exists $R^{\prime}>0$ and $U^{\prime} \subset U$ such that $v_{1}$ and $v_{2}$ do not vanish in $U^{\prime} \cap E_{R^{\prime}}$ since $f_{1}$ and $f_{2}$ are $\rho$-quasiregular. Let $\Gamma_{f_{1}, \rho}$, $\Gamma_{f_{2}, \rho}$ and $\Gamma_{f_{1}, f_{2}, \rho}$ be the following semi-algebraic sets :

$$
\begin{aligned}
& \Gamma_{f_{1}, \rho}=\left\{x \in U \backslash X \mid v_{1}(x)=0\right\}, \quad \Gamma_{f_{2}, \rho}=\left\{x \in U \backslash X \mid v_{2}(x)=0\right\}, \\
& \Gamma_{f_{1}, f_{2}, \rho}=\left\{x \in U \backslash X \mid v_{1}(x) \text { and } v_{2}(x) \text { point in opposite direction }\right\},
\end{aligned}
$$

and let $\Gamma$ be the union of these three sets. Let $r_{0}$ be the greatest critical value of $\rho$ and let $\alpha:] r_{0},+\infty\left[\rightarrow \mathbb{R}\right.$ be defined by $\alpha(r)=\inf \left\{f_{1}(x) \mid x \in \Sigma_{r} \cap \Gamma\right\}$. Then $\alpha$ is a positive semi-algebraic function. To see that it is positive, it is enough to apply Lemma 1.8 of [Dur] to the semi-algebraic subset $X \cap \Sigma_{r}$ of the smooth semi-algebraic set $\Sigma_{r}$. As in Lemma 2.2, this implies that there exists $R>r_{0}$ and an integer $q_{0}$ such that for $x \in \Gamma \cap E_{R}$ and for $q \geq q_{0}$, $(1+\rho(x))^{q} \cdot f_{1}(x)>1$. Since $v_{1 q}=(1+\rho)^{q} \cdot v_{1}$, we see that $\Gamma_{f_{1}, f_{2}, \rho}=\Gamma_{f_{1 q}, f_{2}, \rho}$. We take $\delta_{q}$ to be the minimum of $\delta_{1}$ and 1. This ends the proof of Lemma 4.3 .

Lemma 4.4. For every integer $q \geq 0$, let $f_{1 q}: \mathbb{R}^{n} \rightarrow \mathbb{R}$ (resp. $f_{2 q}: \mathbb{R}^{n} \rightarrow \mathbb{R}$ ) be defined by $f_{1 q}(x)=(1+\rho(x))^{q} \cdot f_{1}(x)$ (resp. $f_{2 q}(x)=(1+\rho(x))^{q} \cdot f_{2}(x)$ ). Let $v_{1 q}$ (resp. $v_{2 q}$ ) be the orthogonal projection of $\nabla f_{1 q}\left(\right.$ resp $\left.\nabla f_{2 q}\right)$ on the levels of $\rho$. There exist $q_{0} \in \mathbb{N}$ and $R>0$ such that for all $q \geq q_{0}$ and for all $l \in \mathbb{N}$ the vector fields $v_{1 q}$ and $v_{2 l}$ are nonzero and do not point in opposite direction in $\left.\left.f_{1 q}^{-1}(] 0, \delta_{q}\right]\right) \cap E_{R}$, where $\delta_{q}$ is a small regular value of $f_{1 q}$ such that $f_{1 q}^{-1}\left(\left[0, \delta_{q}\right]\right) \subset U$ and $f_{1 q}^{-1}\left(\left[0, \delta_{q}\right]\right)$ is closed in $\bar{U}$.

Proof. It is clear because $v_{2 l}=(1+\rho)^{l} \cdot v_{2}$ and $\Gamma_{f_{1 k}, f_{2 l}, \rho}=\Gamma_{f_{1 k}, f_{2}, \rho}$. This ends the proof of Lemma 4.4 .

Let us fix $q$ and $\delta_{q}$ which satisfy the conclusion of Lemma 4.3. Applying Lemma 2.2 to the open semi-algebraic neighborhood $f_{1 q}^{-1}\left(\left[0, \delta_{q}[)\right.\right.$ of $X$ and the approaching function $f_{2}$, we can find $l$ such that $f_{2 l}^{-1}\left(\left[0, \epsilon_{l}\right]\right) \subset$ $f_{1 q}^{-1}\left(\left[0, \delta_{q}[)\right.\right.$, where $\epsilon_{l}$ is a small regular value of $f_{2 l}$. Thanks to Lemma 4.4 , we can proceed as we did for the second case, namely we consider the closed set $S^{\prime}=f_{1 q}^{-1}\left(\left[0, \delta_{q}\right]\right) \backslash f_{2 l}^{-1}\left(\left[0, \epsilon_{l}[)\right.\right.$ and the function $h: S^{\prime} \rightarrow[0,1]$ defined by:

$$
h(x)=\frac{f_{2 l}(x)-\epsilon_{l}}{\left(f_{2 l}(x)-\epsilon_{l}\right)-\left(\delta_{q}-f_{1 q}(x)\right)} .
$$

This ends the proof of Theorem 4.2.

We end this section with this question : if $X$ is a closed non-compact semi-algebraic set in $\mathbb{R}^{n}$, if $\rho_{1}$ and $\rho_{2}$ are two distinct control functions, are a $\rho_{1}$-quasiregular approaching semi-algebraic neighborhood and a $\rho_{2^{-}}$ quasiregular approaching semi-algebraic neighborhood of $X$ isotopic ? 


\section{The SMOOTH CASE}

In this section, we assume that $X$ is a closed non-compact semi-algebraic set in $\mathbb{R}^{n}$ and also a $C^{3}$ submanifold of dimension $k<n$. We also assume that $\rho$ is a control function of class $C^{3}$. We show that any $\rho$-quasiregular approaching semi-algebraic neighborhood of $X$ is isotopic to a tubular neighborhood of $X$. For this, we construct a kind of distance function to $X$ which is $C^{2}$ in a semi-algebraic neighborhood of $X$ and $\rho$-quasiregular.

Let us fix $X$ and $\rho$ satisfying the above assumptions. Let $r_{0}>0$ be such that for all $r \geq r_{0}, \Sigma_{r}$ is a $C^{3}$ submanifold that intersects $X$ transversally. Let $F$ be the following set :

$$
\begin{aligned}
F=\left\{(x, v) \in X \times \mathbb{R}^{n} \mid\right. & \rho(x)>r_{0},\langle v, \nabla \rho(x)\rangle=0 \\
& \text { and } \left.\langle v, w\rangle=0 \text { for all } w \in T_{x}\left(X \cap \Sigma_{\rho(x)}\right)\right\} .
\end{aligned}
$$

It is a $C^{2}$-vector bundle over $X \cap\left\{x \mid \rho(x)>r_{0}\right\}$ whose fibers are $n-k$ dimensional. Moreover it is semi-algebraic. We will denote the fiber over $x$ by $F_{x}$. Observe that $F_{x}$ is the normal space of $X \cap \Sigma_{\rho(x)}$ in $\Sigma_{\rho(x)}$.

Let $N$ be the normal bundle over $X \cap\left\{x \mid \rho(x)<2 r_{0}\right\}$ :

$$
N=\left\{(x, v) \in X \times \mathbb{R}^{n} \mid \rho(x)<2 r_{0} \text { and } v \perp T_{x} X\right\} .
$$

Is is also a $C^{2}$ semi-algebraic vector bundle. We denote the fiber over $x$ by $N_{x}$.

We will glue these two bundles. By [DM] Corollary C.12, it is possible to find a $C^{2}$ semi-algebraic function $\phi: X \mapsto[0,1]$ such that $X \cap E_{7 / 4 r_{0}}=$ $\phi^{-1}(1)$ and $X \cap D_{5 / 4 r_{0}}=\phi^{-1}(0)$. For each $x$ such that $r_{0}<\rho(x)<2 r_{0}$, let $P_{x}$ be restriction to $F_{x}$ of the orthogonal projection to $N_{x}$.

We can define a bundle $H \subset X \times \mathbb{R}^{n}$ over $X$ in the following way :

$$
\begin{aligned}
& \text { if } \rho(x)<5 / 4 r_{0} \text { then } H_{x}=N_{x}, \\
& \text { if } r_{0}<\rho(x)<2 r_{0} \text { then } H_{x}=\left\{v \in \mathbb{R}^{n} \mid \exists w \in F_{x}\right. \\
& \left.\quad \text { such that } v=\phi(x) w+(1-\phi(x)) P_{x}(w)\right\}, \\
& \text { if } \rho(x)>7 / 4 r_{0} \text { then } H_{x}=F_{x} .
\end{aligned}
$$

It is an exercise of linear algebra to prove that $H$ is a vector bundle whose fibres are $n-k$-dimensional planes. Furthermore, it is $C^{2}$ semi-algebraic because $F$ and $N$ are $C^{2}$ semi-algebraic bundles and because $\phi$ is a $C^{2}$ semi-algebraic function. This bundle $H$ will enables us to construct the desired "distance" function to $X$. Let $\varphi$ be the following $C^{2}$ semi-algebraic mapping :

$$
\varphi: \begin{array}{ccc}
H & \rightarrow & \mathbb{R}^{n} \\
(x, v) & \mapsto & x+v .
\end{array}
$$

Then there exists a semi-algebraic open neighborhood $U$ of the zero-section $X \times\{0\}$ in $H$ such that the restriction $\varphi_{\mid U}$ is a $C^{2}$ diffeomorphism onto a neighborhood $V$ of $X$. Moreover, we can take $U$ of the form :

$$
U=\{(x, v) \mid\|v\|<\varepsilon(x)\},
$$


where $\varepsilon$ is a positive $C^{2}$ semi-algebraic function on $X$. The proof of this result is given in [Co2], Lemma 6.15 for the normal bundle. This proof actually holds in our case. This provides us with a $C^{2}$ semi-algebraic retraction $\pi: V \rightarrow X$ and a $C^{2}$ semi-algebraic distance function $d^{\prime}: V \rightarrow X$ defined by $\pi(\varphi(x, v))=x$ and $d^{\prime}(\varphi(x, v))=\|v\|^{2}$.

Lemma 5.1. There exists an open semi-algebraic neighborhood $W$ of $X$ in $V$ such that for every $y \in W, \rho(y) \leq 1,1 \rho(\pi(y))$. Furthermore, one can choose $W$ of the form :

$$
W=\left\{y \in V \mid d^{\prime}(y)<\varepsilon^{\prime}(\pi(y))\right\},
$$

where $\varepsilon^{\prime}: X \rightarrow \mathbb{R}$ is a positive $C^{2}$ semi-algebraic function.

Proof. Let $A$ be the following semi-algebraic set :

$$
A=\{y \in V \mid \rho(y)>1,1 \rho(\pi(y))\} .
$$

Let $\alpha: \pi(A) \rightarrow \mathbb{R}$ be the function defined as follows :

$$
\alpha(x)=\inf \left\{d^{\prime}(y) \mid y \in \pi^{-1}(x) \cap A\right\} .
$$

This is a semi-algebraic function on $\pi(A)$. Let us prove that it is positive. The continuity of $\rho \circ \varphi$ implies that for every $x$ in $\pi(A)$, there exists $\delta_{x}$ with $0<\delta_{x}<\varepsilon(x)$, such that for every $v$ in $H_{x}$ with $\|v\| \leq \delta_{x}, \rho(\varphi(x, v)) \leq$ $1,1 \rho(\varphi(x, 0))$. Since $\|v\|^{2}=d^{\prime}(y)$ if $y=\varphi(x, v)$, this proves that $\alpha(x) \geq$ $\delta_{x}>0$. Let us show that $\alpha$ is locally bounded from below by positive constants, i.e for every $x \in \pi(A)$, there exist $c>0$ and a neighborhood $\Omega$ of $x$ in $\pi(A)$ such that $\alpha>c$ on $\Omega$. If it is not the case, we can find a sequence of points $x_{n}$ in $\pi(A)$ tending to $x$ such that $\alpha\left(x_{n}\right)$ tends to 0 . Hence there exists a sequence of points $y_{n}=\varphi\left(x_{n}, v_{n}\right)$ such that $v_{n}$ tends to $0, x_{n}$ tends to $x$ and $\rho\left(\varphi\left(x_{n}, v_{n}\right)\right)>1,1 \rho\left(\varphi\left(x_{n}, 0\right)\right)$. By continuity, we obtain $\rho(\varphi(x, 0)) \geq 1,1 \rho(\varphi(x, 0))$, which is impossible. Let $\tilde{\alpha}: X \rightarrow \mathbb{R}$ be defined by $\tilde{\alpha}(x)=\alpha(x)$ if $x \in \pi(A)$ and $\tilde{\alpha}(x)=\varepsilon(x)$ if $x \notin \pi(A)$. The function $\tilde{\alpha}$ is semi-algebraic, positive and locally bounded from below by positive constants. Applying Lemma 6.12 of [Co2], we can find a positive semi-algebraic $C^{2}$ function $\varepsilon^{\prime}: X \rightarrow \mathbb{R}$ such that $\varepsilon^{\prime}<\tilde{\alpha}$ on $X$.

Let us study the function $d^{\prime}: W \rightarrow \mathbb{R}$ more precisely. Let $B$ be the following semi-algebraic set :

$$
B=\left\{y \in W \cap E_{2 r_{0}} \mid \frac{\langle\nabla \rho(y), \nabla \rho(\pi(y))\rangle}{\|\nabla \rho(y)\| \cdot\|\nabla \rho(\pi(y))\|}<0,9\right\} .
$$

Let $\beta: \pi(B) \rightarrow \mathbb{R}$ be the function defined as follows :

$$
\beta(x)=\inf \left\{d^{\prime}(y) \mid y \in \pi^{-1}(x) \cap B\right\} .
$$

This is a semi-algebraic function on $\pi(B)$ and for every $x \in \pi(B), \beta(x) \leq$ $\varepsilon^{\prime}(x)$. The same argument as in the above lemma shows that $\beta$ is positive and locally bounded from below by positive constants. Let $\tilde{\beta}: X \rightarrow \mathbb{R}$ be defined by $\tilde{\beta}(x)=\beta(x)$ if $x \in \pi(B)$ and $\tilde{\beta}(x)=\varepsilon^{\prime}(x)$ if $x \notin \pi(B)$. The function $\tilde{\beta}$ is semi-algebraic, positive and locally bounded from below 
by positive constants. We can find a positive semi-algebraic $C^{2}$ function $\varepsilon ": X \rightarrow \mathbb{R}$ such that $\varepsilon "<\tilde{\beta}$ on $X$.

Let $W^{\prime}$ be defined by :

$$
W^{\prime}=\left\{y \in V \mid d^{\prime}(y)<\varepsilon^{\prime \prime}(\pi(y))\right\} .
$$

Note that $W^{\prime}$ is included in $W$. For every $y$ in $W^{\prime} \cap E_{2 r_{0}}$, we have :

$$
\frac{\langle\nabla \rho(y), \nabla \rho(\pi(y))\rangle}{\|\nabla \rho(y)\| \cdot\|\nabla \rho(\pi(y))\|} \geq 0,9 .
$$

Since $\nabla d^{\prime}(y)$ belongs to $[\nabla \rho(\pi(y))]^{\perp}$, this can be reformulated in the following way : for every $y$ in $W^{\prime} \cap E_{2 r_{0}}$, we have :

$$
\frac{\left\langle\nabla \rho(y), \nabla d^{\prime}(y)\right\rangle}{\|\nabla \rho(y)\| \cdot\left\|\nabla d^{\prime}(y)\right\|} \leq \sqrt{0,19} .
$$

Lemma 5.2. There exist $q_{0} \in \mathbb{N}$ and $r_{0}^{\prime}>0$ such that for every $q \geq q_{0}$ and for every $x \in X \cap E_{r_{0}^{\prime}}$,

$$
\frac{1}{(1+\rho(x))^{q}} \leq \varepsilon^{\prime \prime}(x) .
$$

Proof. Let $h:[0,+\infty[\rightarrow \mathbb{R}$ be defined by :

$$
h(r)=\min \left\{\varepsilon^{\prime \prime}(x) \mid x \in X \cap \Sigma_{r}\right\} .
$$

Since $h$ is a positive semi-algebraic function, there exists an integer $q_{0}$ and a real $r_{0}^{\prime}>0$ such that $\frac{1}{h(r)}<r^{q_{0}}$ for every $r \geq r_{0}^{\prime}$. Hence for every $q \geq q_{0}$ and every $x \in X \cap E_{r_{0}^{\prime}}$, we have :

$$
\frac{1}{(1+\rho(x))^{q}} \leq \varepsilon^{\prime \prime}(x) .
$$

Corollary 5.3. There exist $q_{0} \in \mathbb{N}$ and $r_{0} ">0$ such that for every $q \geq q_{0}$ and for every $y \in W^{\prime} \cap E_{r_{0} \text { ", }}$,

$$
\frac{1}{(1+\rho(\pi(y)))^{q}} \leq \varepsilon^{\prime \prime}(\pi(y)) .
$$

Proof. By Lemma 5.1, we can find $r_{0} ">0$ such that $\pi(y)$ belongs to $X \cap E_{r_{0}^{\prime}}$ if $y$ belongs to $W^{\prime} \cap E_{r_{0}}$.

Lemma 5.4. There exist $q_{1} \in \mathbb{N}$ and $r_{1}^{\prime}>0$ such that for every $q \geq q_{1}$ and for every $x \in X \cap E_{r_{1}^{\prime}},\|\nabla \rho(x)\| \leq(1+\rho(x))^{q}$.

Proof. Let $c>0$ be such that $[c,+\infty[$ does not contain any critical value of $\rho$. Let $l:[c,+\infty[\rightarrow \mathbb{R}$ be defined by :

$$
l(r)=\max \left\{\|\nabla \rho(x)\| \mid x \in X \cap \Sigma_{r}\right\} .
$$

Since $l$ is a positive semi-algebraic function, there exits an integer $q_{1}$ and a real $r_{1}^{\prime}>0$ such that $l(r)<r^{q_{1}}$ for every $r \geq r_{1}^{\prime}$. Hence for every $q \geq q_{1}$ and every $x \in X \cap E_{r_{1}^{\prime}}$, we have $\|\nabla \rho(x)\| \leq(1+\rho(x))^{q}$. 
Corollary 5.5. There exist $q_{1} \in \mathbb{N}$ and $r_{1} ">0$ such that for every $q \geq q_{1}$ and for every $y \in W^{\prime} \cap E_{r_{1}}$, $\|\nabla \rho(\pi(y))\| \leq(1+\rho(\pi(y)))^{q}$.

Proof. The proof is the same as Corollary 5.3.

Proposition 5.6. There exists an integer $q_{2}$ such that for every $q \geq q_{2}$, the function $d_{q}^{\prime}: W^{\prime} \rightarrow \mathbb{R}$ defined by $d_{q}^{\prime}(y)=\left(1+\rho(\pi(y))^{q} . d^{\prime}(y)\right.$ is a $\rho$ quasiregular approaching function for $X$ in $W^{\prime}$.

Proof. Since $W^{\prime}=\left\{y \in V \mid d^{\prime}(y)<\varepsilon^{\prime \prime}(\pi(y))\right\}$ and $\varepsilon^{\prime \prime}$ is a positive function, $\left(X, W^{\prime}, d^{\prime}\right)$ satisfies the condition $(A)$. Let

$$
W_{1}=\left\{y \in V \mid d^{\prime}(y)<\frac{1}{2} \varepsilon^{\prime \prime}(\pi(y))\right\} .
$$

We have $\overline{W_{1}} \subset W^{\prime}$. By Corollary 5.3, for every $q \geq q_{0}$, the set $E_{r_{0}}$ " $\cap$ $d_{q}^{-1}\left(\left[0, \frac{1}{4}\right]\right)$ is included in $W_{1}$. The tuple $\left(X, W^{\prime}, d_{q}^{\prime}\right)$ satisfies the condition $(A)$. As it has been already explained in Lemma 2.2, there exists $\epsilon_{q}>0$ such that $d_{q}^{-1}\left(\left[0, \epsilon_{q}\right]\right) \cap D_{r_{0}}$ " $\subset W_{1} \cap D_{r_{0}}$. . Let $\delta_{q}$ be the minimum of $\frac{1}{4}$ and $\epsilon_{q}$. The set $d_{q}^{\prime-1}\left(\left[0, \delta_{q}\right]\right)$ is included in $W_{1}$, hence closed in $\overline{W_{1}}$ and in $\overline{W^{\prime}}$. This proves that $d_{q}^{\prime}$ is an approaching function for $X$ in $W^{\prime}$.

Let us show that it is $\rho$-quasiregular. Let us fix $r$ greater than $r_{0}$ ", $r_{1}$ " and $2 r_{0}$ and let us fix $q_{2}$ greater than $q_{0}$ and $q_{1}$. For every $y$ in $W \cap E_{r}$, let $P_{y}$ be the orthogonal projection onto the space $\nabla \rho(y)^{\perp}$. We have :

$$
\nabla d_{q}^{\prime}(y)=(1+\rho(\pi(y)))^{q-1} \cdot\left[(1+\rho(\pi(y))) \nabla d^{\prime}(y)+q d^{\prime}(y) \nabla \rho(\pi(y))\right],
$$

hence,

$$
\frac{P_{y}\left(\nabla d_{q}^{\prime}(y)\right)}{(1+\rho(\pi(y)))^{q-1}}=(1+\rho(\pi(y))) P_{y}\left(\nabla d^{\prime}(y)\right)+q d^{\prime}(y) P_{y}(\nabla \rho(\pi(y))) .
$$

Let us prove that, for $q \geq q_{2}$ and $R \geq r$ sufficiently big, $T(y)$ can not vanish if $y$ belongs to $\left.\left.d_{q}^{\prime-1}(] 0,1\right]\right) \cap E_{R}$, where

$$
T(y)=(1+\rho(\pi(y))) P_{y}\left(\nabla d^{\prime}(y)\right)+q d^{\prime}(y) P_{y}(\nabla \rho(\pi(y))) .
$$

First observe that if $y$ lies in $d_{q}^{\prime-1}([0,1]) \cap E_{R}, q \geq q_{2}$ and $R \geq r$, then :

and

$$
\frac{\langle\nabla \rho(y), \nabla \rho(\pi(y))\rangle}{\|\nabla \rho(y)\| \cdot\|\nabla \rho(\pi(y))\|} \geq 0,9
$$

This implies that

$$
\frac{\left\langle\nabla \rho(y), \nabla d^{\prime}(y)\right\rangle}{\|\nabla \rho(y)\| \cdot\left\|\nabla d^{\prime}(y)\right\|} \leq \sqrt{0,19}
$$

$$
\left\|P_{y}(\nabla \rho(\pi(y)))\right\| \leq \sqrt{0,19}\|\nabla \rho(\pi(y))\|
$$

and

$$
\left\|P_{y}\left(\nabla d^{\prime}(y)\right)\right\| \geq 0,9\left\|\nabla d^{\prime}(y)\right\| .
$$

Therefore, we have :

$$
\left\|q d^{\prime}(y) P_{y}(\nabla \rho(\pi(y)))\right\| \leq \sqrt{0,19} q d^{\prime}(y)\|\nabla \rho(\pi(y))\|,
$$


and,

$$
\left\|(1+\rho(\pi(y))) P_{y}\left(\nabla d^{\prime}(y)\right)\right\| \geq 0,9(1+\rho(\pi(y))) \cdot\left\|\nabla d^{\prime}(y)\right\|,
$$

that is to say :

$$
\left\|(1+\rho(\pi(y))) P_{y}\left(\nabla d^{\prime}(y)\right)\right\| \geq 0,9(1+\rho(\pi(y))) \cdot 2 \sqrt{d^{\prime}(y)} .
$$

In order to prove that $T(y)$ does not vanish if $y \in d_{q}^{\prime-1}([0,1]) \cap E_{R}$ for $q \geq q_{2}$ and $R \geq r$ sufficiently big, it is enough to prove that :

$$
\frac{1,8}{\sqrt{0,19}}>\frac{q \sqrt{d^{\prime}(y)}\|\nabla \rho(\pi(y))\|}{1+\rho(\pi(y))} .
$$

But if $\left.\left.y \in d_{q}^{\prime-1}(] 0,1\right]\right) \cap E_{R}$ where $q \geq q_{2}$ and $R \geq r$ then we have :

$$
\sqrt{d^{\prime}(y)} \leq \frac{1}{(1+\rho(\pi(y)))^{\frac{q}{2}}} .
$$

So, if we show that

$$
\frac{1,8}{\sqrt{0,19}}>\frac{q\|\nabla \rho(\pi(y))\|}{(1+\rho(\pi(y)))^{\frac{q}{2}+1}}
$$

then the required result is established. Let $q$ be such that $\frac{q}{2}+1>q_{1}$. By Corollary 5.5, we have :

$$
\frac{q\|\nabla \rho(\pi(y))\|}{(1+\rho(\pi(y)))^{\frac{q}{2}+1}} \leq \frac{q}{(1+\rho(\pi(y)))^{\frac{q}{2}+1-q_{1}}}
$$

for $y \in d_{q}^{\prime-1}([0,1]) \cap E_{R}, R \geq r$. Lemma 5.1 implies that there exists $R_{q} \geq r$ such that if $y$ belongs to $d_{q}^{\prime-1}([0,1]) \cap E_{R}$, with $R \geq R_{q}$, then we have :

$$
\frac{q}{(1+\rho(\pi(y)))^{\frac{q}{2}+1-q_{1}}}<\frac{1,8}{\sqrt{0,19}} .
$$

This proves the proposition.

We can state the main result of this section.

Theorem 5.7. Let $X$ be a closed non-compact semi-algebraic set in $\mathbb{R}^{n}$ which is a $C^{3}$ submanifold. Let $\rho$ be a control function of class $C^{3}$. Any $\rho$-quasiregular approaching semi-algebraic neighborhood of $X$ is isotopic to a tubular neighborhood of $X$.

Proof. By the previous proposition, we known that there exist $\rho$-quasiregular approaching functions $d_{q}^{\prime}$ for $X$ in $W$ of the form $d_{q}^{\prime}(y)=(1+\rho(\pi(y)))^{q}$. $d^{\prime}(y)$. But for $\nu>0$ sufficiently small the set $d_{q}^{\prime-1}([0, \nu])$ is a tubular neighborhood of $X$. It is enough to use Theorem 4.2 to conclude. 


\section{Degree formulas for the Euler-Poincaré Characteristic of}

A CLOSED SEMI-ALGEBRAIC SET

In this section, we give degree formulas for the Euler-Poincaré characteristic of a closed semi-algebraic set $X$ included in $\mathbb{R}^{n}$. When $X$ is algebraic, we deduce from these formulas a Petrovskii-Oleinik inequality for $|1-\chi(X)|$.

Let $X \subset \mathbb{R}^{n}$ be a closed semi-algebraic set and let $f: \mathbb{R}^{n} \rightarrow \mathbb{R}$ be a nonnegative $C^{2}$ semi-algebraic function such that $X=f^{-1}(0)$, i.e $f$ is an approaching function for $X$ in $\mathbb{R}^{n}$. Let $\rho$ be a control function. For every $q \in \mathbb{N}$, we will denote by $f_{q}$ the function defined by $f_{q}(x)=(1+\rho(x))^{q} \cdot f(x)$. We will also denote by $\Gamma_{f, \rho}\left(\right.$ resp. $\left.\Gamma_{f_{q}, \rho}\right)$ the following polar set :

$$
\Gamma_{f, \rho}=\left\{x \in \mathbb{R}^{n} \backslash X \mid \nabla f(x)\left(\text { resp. } \nabla f_{q}(x)\right) \text { and } \nabla \rho(x) \text { are colinear }\right\} .
$$

Note that $\Gamma_{f, \rho}=\Gamma_{f_{q}, \rho}$ for each $q \in \mathbb{N}$. The following proposition is similar to Proposition 2.7 and is proved in the same way.

Proposition 6.1. There exists an integer $q_{0}$ such that for every $q \geq q_{0}$, the following property holds : for any sequence $\left(x_{k}\right)_{k \in \mathbb{N}} \subset \Gamma_{f_{q}, \rho}$ such that $\lim _{k \rightarrow+\infty}\left\|x_{k}\right\|=+\infty$, we have $\lim _{k \rightarrow+\infty} f_{q}\left(x_{k}\right)=+\infty$.

Let us fix an integer $q$ satisfying the property of the previous proposition. Let $\Sigma\left(f_{q}\right)$ be the set of critical points of $f_{q}$ and let $\Sigma^{*}\left(f_{q}\right)$ be the set of critical points of $f_{q}$ lying in $\mathbb{R}^{n} \backslash X$.

Corollary 6.2. The set $\Sigma^{*}\left(f_{q}\right)$ is compact.

Proof. It is clearly closed as the set of preimages of the nonzero critical values of $f_{q}$. If it is not bounded, there exists a sequence of points $\left(x_{k}\right)_{k \in \mathbb{N}}$ such that $x_{k} \notin X, \nabla f_{q}\left(x_{k}\right)=0$ and $\lim _{k \rightarrow+\infty}\left\|x_{k}\right\|=+\infty$. Since for each $k \in \mathbb{N}, x_{k}$ also belongs to $\Gamma_{f_{q}, \rho}$, this gives a contradiction.

Let us decompose $\Sigma^{*}\left(f_{q}\right)$ into the finite union of its connected components $K_{1}^{q}, \ldots, K_{m_{q}}^{q}$ :

$$
\Sigma^{*}\left(f_{q}\right)=\bigcup_{i=1}^{m_{q}} K_{i}^{q} .
$$

Before stating the main results of this section, we need to introduce some notations. For each $i \in\left\{1, \ldots, m_{q}\right\}$, let $U_{i}$ be a relatively compact neighborhood of $K_{i}^{q}$ such that $\partial U_{i}$ is a smooth hypersurface and $U_{i} \cap \Sigma^{*}\left(f_{q}\right)=K_{i}^{q}$. For any mapping $F: \mathbb{R}^{n} \rightarrow \mathbb{R}^{n}$ such that $F^{-1}(0) \cap U_{i}=K_{i}^{q}$ or $F^{-1}(0) \cap U_{i}$ is empty, we will denote by $\operatorname{deg}_{K_{i}^{q}} F$ the topological degree of the following mapping :

$$
\begin{aligned}
& \frac{F}{\|F\|} \quad: \quad \partial U_{i} \quad \rightarrow \quad S^{n-1} \\
& x \mapsto \frac{F(x)}{\|F(x)\|} .
\end{aligned}
$$

It is well-known that this topological degree does not depend on the choice of the relatively compact neighborhood $U_{i}$. 
Theorem 6.3. The Euler-Poincaré characteristic of $X$ is related to $\nabla f_{q}$ by the following formula:

$$
\chi(X)=1-\sum_{i=1}^{m_{q}} \operatorname{deg}_{K_{i}^{q}} \nabla f_{q} .
$$

Proof. By Proposition 6.1, $f_{q}$ is a $\rho$-quasiregular approaching function for $X$ in $\mathbb{R}^{n}$. Theorem 3.2 implies that for $\varepsilon>0$ sufficiently small :

$$
\chi(X)=\chi\left(\left\{f_{q} \leq \varepsilon\right\}\right) .
$$

By the Mayer-Vietoris sequence, we have :

$$
1=\chi\left(\left\{f_{q} \leq \varepsilon\right\}\right)+\chi\left(\left\{f_{q} \geq \varepsilon\right\}\right)-\chi\left(\left\{f_{q}=\varepsilon\right\}\right) .
$$

We will apply Morse theory to the manifold with boundary $D_{R}$ and to the function $f_{q}$. We will follow the terminology of [Dut], Section 2, p.4647. Let us first show that $f_{q}$ does not admit any inward critical point on $\Sigma_{R} \cap\left\{f_{q} \geq \varepsilon\right\}$ for $R$ sufficiently big and $\varepsilon$ sufficiently small (an inward critical point $p$ is a point critical point $p$ of $f_{q_{\mid \Sigma_{R}}}$ such that $\nabla f_{q}(p)$ is a negative multiple of $\nabla \rho(p))$. If it is not the case, then we can find a sequence of points $\left(x_{k}\right)_{k \in \mathbb{N}}$ in $\Gamma_{f_{q}, \rho}$ such that $\nabla f_{q}\left(x_{k}\right)$ is a negative multiple of $\nabla \rho\left(x_{k}\right)$. Using the version at infinity of the Curve Selection Lemma (see [NZ], Lemma 2), we obtain that $\lim _{k \rightarrow+\infty} f_{q}\left(x_{k}\right)$ exists and belongs to $[0,+\infty[$, which contradicts the property of Proposition 6.1.

Let us fix $R$ sufficiently big and $\varepsilon$ sufficiently small so that $\Sigma^{*}\left(f_{q}\right) \subset D_{R}$, $f_{q}$ does not have inward critical points in $\Sigma_{R} \cap\left\{f_{q} \geq \varepsilon\right\}$ and

$$
\chi\left(\left\{f_{q} \geq \varepsilon\right\}\right)=\chi\left(\left\{f_{q} \geq \varepsilon\right\} \cap D_{R}\right) \text { and } \chi\left(\left\{f_{q}=\varepsilon\right\}\right)=\chi\left(\left\{f_{q}=\varepsilon\right\} \cap D_{R}\right) .
$$

Since $f_{q}$ does not have inward critical points in $\Sigma_{R} \cap\left\{f_{q} \geq \varepsilon\right\}$, Morse theory for manifolds with boundary implies that

$$
\chi\left(\left\{f_{q} \geq \varepsilon\right\} \cap D_{r}\right)-\chi\left(\left\{f_{q}=\varepsilon\right\} \cap D_{r}\right)=\sum_{i=1}^{m_{q}} \operatorname{deg}_{K_{i}^{q}} \nabla f_{q} .
$$

The final result is just a combination of equality (1) and equality (2).

Let $F_{q}: \mathbb{R}^{n} \rightarrow \mathbb{R}^{n}$ be the mapping defined by $F_{q}(x)=q \cdot f \cdot \nabla \rho+(1+\rho) \cdot \nabla f$. Note that for every $x \in \mathbb{R}^{n}, \nabla f_{q}(x)=(1+\rho(x))^{q-1} \cdot F_{q}(x)$. Hence $\nabla f_{q}$ and $F_{q}$ admit the same zeros in $\mathbb{R}^{n}$.

Corollary 6.4. The Euler-Poincaré characteristic of $X$ is related to $F_{q}$ by the following formula:

$$
\chi(X)=1-\sum_{i=1}^{m^{q}} \operatorname{deg}_{K_{i}^{q}} F_{q} .
$$

Proof. It is enough to prove that for every $i \in\left\{1, \ldots, m_{q}\right\}, \operatorname{deg}_{K^{q}} F_{q}=$ $\operatorname{deg}_{K_{i}^{q}} \nabla f_{q}$. Let us choose a relatively compact neighborhood $U_{i}$ of $K_{i}^{q}$ such that $\partial U_{i}$ is a smooth manifold, $F_{q}^{-1}(0) \cap U_{i}=K_{i}^{q}=\nabla f_{q}^{-1}(0) \cap U_{i}$. The result is clear since on $\partial U_{i}$, we have $\frac{\nabla f_{q}}{\left\|\nabla f_{q}\right\|}=\frac{F_{q}}{\left\|F_{q}\right\|}$. 
Corollary 6.5. Let $G_{q}: \mathbb{R}^{n+1} \rightarrow \mathbb{R}^{n+1}$ be the mapping defined by $G_{q}(\lambda ; x)=$ $\left(f(x) \lambda-1, F_{q}(x)\right)$. The set $G_{q}^{-1}(0)$ is compact and if $R>0$ is such that $G_{q}^{-1}(0) \subsetneq B_{R}^{n+1}$, then

$$
\chi(X)=1-\operatorname{deg}_{S_{R}^{n}} G_{q} .
$$

Here $B_{R}^{n+1}$ and $S_{R}^{n}$ are the ball and the sphere of radius $R$ in $\mathbb{R}^{n+1}$.

Proof. Since $G_{q}(\lambda ; x)=0$ if and only if $F_{q}(x)=0, f(x) \neq 0$ and $\lambda=\frac{1}{f(x)}$, it is straightforward to see that $G_{q}^{-1}(0)$ is compact. The rest of the proof is easy.

These formulas are global versions of a result due to Khimshiasvili [Khi] on the Euler characteristic of the real Milnor fibre. It states that, if $g$ : $\left(\mathbb{R}^{n}, 0\right) \rightarrow(\mathbb{R}, 0)$ is an analytic function-germ with an isolated critical point at the origin, then

$$
\chi\left(g^{-1}(\delta) \cap B_{\varepsilon}^{n}\right)=1-\operatorname{sign}(-\delta)^{n} \operatorname{deg}_{0} \nabla g,
$$

for any regular value $\delta$ of $g, 0<|\delta| \ll \varepsilon \ll 1$. Here $\operatorname{deg}_{0} \nabla g$ is the topological degree of $\frac{\nabla g}{\|\nabla g\|}: S_{\varepsilon}^{n-1} \rightarrow S^{n-1}$.

In their fundamental paper $[\mathrm{OP}]$, Petrovskii and Oleinik estimated the Euler characteristic of some real projective algebraic sets. More precisely they gave an upper bound for the following quantities :

- $|\chi(Y)-1|$ where $Y$ is a real projective hypersurface of even dimension,

- $\left|2 \chi\left(Z_{-}\right)-1\right|$ where $Z_{-}$is the subset of $\mathbb{R} P^{n}$ that is bounded by a real projective hypersurface $Y$ of odd dimension and even degree and corresponds to the negative values of the polynomial that determines $Y$.

These results were generalized by Kharlamov [Kha1,Kha2]. In [Ar], Arnol'd found a new proof based, on Khimshiashvili's formula, and an equivalent formulation of the original Petrovskii-Oleinik inequalities. Let us state Arnol'd's version of these inequalities. We need some notations. With every $n$-tuples of positive integers $\mathbf{m}=\left(m_{1}, \ldots, m_{n}\right)$ and with every positive integer $m_{0}$, we will associate the following objects :

- $\Delta_{n}(\mathbf{m})$ is the parallelepiped in $\mathbb{R}^{n}$ defined by the inequalities

$$
0 \leq x_{1} \leq m_{1}-1, \ldots, 0 \leq x_{n} \leq m_{n}-1,
$$

- $\mu=m_{1} \cdots m_{n}$ is the number of integral points in the parallelepiped $\Delta_{n}(\mathbf{m})$

- $\nu=\frac{1}{2}\left(m_{1}+\cdots+m_{n}-n\right)$ is the mean value of the sum of the coordinates of the points in $\Delta_{n}(\mathbf{m})$,

- $\Pi_{n}(\mathbf{m})$ is the number of integral points on the central section $x_{1}+$ $\cdots+x_{n}=\nu$ of the parallelepiped $\Delta_{n}(\mathbf{m})$,

- $\Pi_{n}\left(\mathbf{m}, m_{0}\right)$ is the number of integral points in $\Delta_{n}(\mathbf{m})$ that lie in the strip

$$
\nu-\frac{1}{2} m_{0} \leq x_{1}+\cdots+x_{n} \leq \nu+\frac{1}{2} m_{0},
$$


- $O_{n}\left(\mathbf{m}, m_{0}\right)$ is the number of integral points in $\Delta_{n}(\mathbf{m})$ that satisfy the inequalities

$$
\nu-\frac{1}{2} m_{0} \leq x_{1}+\cdots+x_{n} \leq \nu .
$$

Arnol'd [Ar] proved the following theorem.

Theorem 6.6. Let $f$ be a homogeneous polynomial of degree $d$ in $\mathbb{R}^{n}$ defining a non-singular hypersurface $Y$ in $\mathbb{R} P^{n-1}$. If $n$ is even, we have:

$$
|1-\chi(Y)| \leq \Pi_{n}(\mathbf{d}-\mathbf{1}) \text {, where } \mathbf{d}-\mathbf{1}=(d-1, \ldots, d-1) \text { in } \mathbb{N}^{n} .
$$

If $n$ is odd and $d$ is even, let $Z_{-}$be the subset of $\mathbb{R} P^{n}$ that is bounded by $Y$ and corresponds to the negative values of the polynomial $f$. We have:

$$
\left|1-2 \chi\left(Z^{-}\right)\right| \leq \Pi_{n}(\mathbf{d}-\mathbf{1}) .
$$

In [Kho1] (see also [Kho2]), Khovanskii gave an affine version of this theorem.

Proposition 6.7. Let $f: \mathbb{R}^{n} \rightarrow \mathbb{R}$ be a polynomial of degree $d$ such that the surface $\{f=0\}$ is nonsingular and the domains $\{f \leq c\}$ are compact for every $c \in \mathbb{R}$. Then the Euler-Poincaré of the domain $\{f \leq 0\}$ satisfies the inequality

$$
|1-2 \chi(\{f \leq 0\})| \leq \Pi_{n}(\mathbf{d}-\mathbf{1}, d-1),
$$

where $\mathbf{d}-\mathbf{1}=(d-1, \ldots, d-1)$ in $\mathbb{N}^{n}$.

Our aim is to give a Petroskii-Oleinik inequality for the Euler-Poincaré characteristic of any algebraic set in $\mathbb{R}^{n}$. Let $X$ be an algebraic set in $\mathbb{R}^{n}$ defined as the zero set of the polynomials $f_{1}, \ldots, f_{k}$, each $f_{i}$ having degree $d_{i}$. Hence $X=\left\{x \in \mathbb{R}^{n} \mid f(x)=0\right\}$ where $f=f_{1}^{2}+\cdots+f_{k}^{2}$. The degree of the polynomial $f$ is $d=2 \operatorname{Max}\left\{d_{1}, \ldots, d_{k}\right\}$. The following proposition gives an upper bound for $|1-\chi(X)|$ in terms of $d$.

Proposition 6.8. Let $X$ be an algebraic set in $\mathbb{R}^{n}$ defined as the set of zeros of a nonnegative polynomial $f$ of even degree $d$. We have:

$$
|1-\chi(X)| \leq O_{n+1}(\mathbf{d}+\mathbf{1}, 2),
$$

where $\mathbf{d}+\mathbf{1}=(d+1, \ldots, d+1)$ in $\mathbb{N}^{n+1}$.

Proof. Let $\omega: \mathbb{R}^{n} \rightarrow \mathbb{R}$ be defined by $\omega(x)=x_{1}^{2}+\ldots+x_{n}^{2}$. Applying the argument described above to the functions $f$ and $\omega$, we find that there exists an integer $q$ sufficiently big and a real $R>0$ sufficiently big such that:

$$
\chi(X)=1-\operatorname{deg}_{S_{R}^{n}} G_{q} .
$$

Let $\delta$ be a small positive regular value of $G_{q}$ and let $\left\{p_{1}, \ldots, p_{l}\right\}$ be the set of preimages of $\delta$ by $G_{q}$ lying in $S_{R}^{n}$. We have :

$$
1-\chi(X)=\operatorname{deg}_{S_{R}^{n}}\left(G_{q}-\delta\right)=\sum_{j=1}^{l} \operatorname{deg}_{p_{j}}\left(G_{q}-\delta\right) .
$$


Since each component of $G_{q}-\delta$ has a degree not exceeding $d+1$, the square of the euclidian distance function in $\mathbb{R}^{n+1}$ has degree 2 and $2+(n+1)(d+1) \equiv$ $n+1 \bmod 2$, Theorem 2 of [Kho1] applied to the vector field $G_{q}-\delta$ and the function $R-\left(x_{1}^{2}+\cdots+x_{n}^{2}+\lambda^{2}\right)$ gives :

$$
\left|\sum_{j=1}^{l} \operatorname{deg}_{p_{j}}\left(G_{q}-\delta\right)\right| \leq O_{n+1}(\mathbf{d}+\mathbf{1}, 2),
$$

where $\mathbf{d}+\mathbf{1}=(d+1, \ldots, d+1)$ in $\mathbb{N}^{n+1}$.

\section{REFERENCES}

[Ar] ARNOLD, V.I. : Index of a singular point of a vector field, the Petrovski-Oleinik inequality, and mixed Hodge structures, Funct. Anal. and its Appli. 12 (1978), $1-14$.

[BCR] BOCHNAK, J., COSTE, M., ROY, M.F. : Géométrie algébrique réelle, Ergebnisse der Mathematik 12 Springer-Verlag 1987.

[Br] BROUGHTON, S.A. : On the topology of polynomial hypersurfaces. Singularities, Part 1 (Arcata, Calif., 1981), 167-178, Proc. Sympos. Pure Math., 40, Amer. Math. Soc., Providence, RI, 1983.

[Co1] COSTE, M : An introduction to semi-algebraic geometry, Dottorato di Recerca in Matematica, Dip. Mat. Univ. Pisa. Istituti Editoriali e Poligrafici Internazionali, Pisa (2000).

[Co2] COSTE, M. : An introduction to o-minimal geometry., Dottorato di Recerca in Matematica, Dip. Mat. Univ. Pisa. Istituti Editoriali e Poligrafici Internazionali, Pisa (2000).

[DM] Van den DRIES, L., MILLER, C. : Geometric categories and o-minimal structures, Duke Math. J., 84 (1996), no. 2, 497-540.

[Dur] DURFEE, A.H. : Neighborhoods of algebraic sets, Trans. Am. Math. Soc. 276 (1983), no. 2 , 517-530.

[Dut] DUTERTRE, N. : Geometrical and topological properties of real polynomial fibres, Geometriae Dedicata 105 (2004), no. 1, 43-59.

[Fe] FEKAK, A. : Exposants de Lojasiewicz pour les fonctions semi-algébriques, Ann. Polon. Math. 56 (1992), no. 2, 123-131.

[Kha1] KHARLAMOV, V. M. : A generalized Petrovskii inequality, Funkcional. Anal. i Prilovzen. 8 (1974), no. 2, 50-56.

[Kha2] KHARLAMOV, V. M. : A generalized Petrovskii inequality II, Funkcional. Anal. i Prilovzen. 9 (1975), no. 3, 93-94.

[Khi] KHIMSHIASHVILI, G.M. : On the local degree of a smooth map, Soobshch. Akad. Nauk Gruz. SSR 85 (1977), 309-311.

[Kho1] KHOVANSKII, A.G. : Index of a polynomial vector field, Functional Anal. Appl. 13 (1978), No. 1, 38-45.

[Kho2] KHOVANSKII, A.G. : Boundary indices of polynomial 1-forms with homogeneous components, St. Petersburg Math. J. 10 (1999), no. 3, 553-575.

[Ku] KURDYKA, K. : On gradients of functions definable in o-minimal structures, Ann. Inst. Fourier 48 (1998), no. 3, 769-783.

[KMP] KURDYKA, K., MOSTOWSKI, T., PARUSINSKI, A. : Proof of the gradient conjecture of R. Thom, Ann. of Math. (2) 152 (2000), no. 3, 763-792.

[KP] KURDYKA, K., PARUSINSKI, A. : $w_{f}$-stratification of subanalytic functions and the Lojasiewicz inequality, C. R. Acad. Sci. Paris Sr. I Math. 318 (1994), no. $2,129-133$. 
[Lo1] LOJASIEWICZ, S. : Une propriété topologique des sous-ensembles analytiques réels, Colloques Internationaux du CNRS, Les équations aux dérivées partielles, 117, ed. B. Malgrange (Paris 1962), Publications du CNRS, Paris, 1963.

[Lo2] LOJASIEWICZ, S. : Sur les trajectoires du gradient d'une fonction analytique réelle, Seminari di Geometria 1982-83, Bologna, 1984, 115-117.

[NS] NOWEL, A., SZAFRANIEC, Z. : On trajectories of analytic gradient vector fields, J. Differential Equations 184 (2002), no. 1, 215-223.

[NZ] NEMETHI, A., ZAHARIA, A.: Milnor fibration at infinity, Indag. Math. 3 (1992), 323-335.

[OP] OLEINIK, O.A., PETROVSKII, I.G. : On the topology of real algebraic surfaces, Amer. Math. Soc. Trans., 70, Amer. Math. Soc., Providence, RI (1952).

[Ti] TIBAR, M. : Regularity at infinity of real and complex polynomial functions. Singularity theory (Liverpool, 1996), 249-264, London Math. Soc. Lecture Note Ser., 263, Cambridge Univ. Press, Cambridge, 1999.

Université de Provence, Centre de Mathématiques et Informatique, 39 rue Joliot-Curie, 13453 Marseille Cedex 13, France.

E-mail address: dutertre@cmi.univ-mrs.fr 